\title{
Three-point current correlation functions as probes of effective conformal theories
}

\author{
Kassahun Betre ${ }^{1}$ \\ SLAC National Accelerator Laboratory, \\ Menlo Park, CA 94025 U.S.A. \\ E-mail: kassahun@stanford.edu
}

ABSTRACT: The first sub-leading, order $1 / \sqrt{\lambda}$ correction to the three-point current correlation function $\left\langle J_{i}^{a}(x) J_{j}^{b}(y) J_{k}^{c}(z)\right\rangle$ of a strongly coupled conformal system with non-Abelian global symmetry is shown to come uniquely from the non-renormalizable bulk operator $\left(F_{\mu \nu}\right)^{3}$. The non-renormalizable correction is suppressed by powers of the cutoff scale $\Lambda$ of the bulk effective theory, which corresponds to a dimension cutoff $\Delta=R_{\text {AdS }} \Lambda$ in the boundary effective conformal theory. The contribution of the non-renormalizable term to the three-point function is calculated from the weakly coupled AdS dual in the large $N$ limit. It is shown to have a polarization structure different from the leading contribution, which comes from the renormalizable $\left(F_{\mu \nu}\right)^{2}$ operator. This suggests a possible experimental probe of the effective conformal description through a measurement of the cutoff dimension $\Delta$ in strongly coupled condensed matter systems.

KEYWORDs: 1/N Expansion, Holography and condensed matter physics (AdS/CMT), Strong Coupling Expansion

ARXIV EPRINT: 1311.2090

\footnotetext{
${ }^{1}$ Work supported by the US Department of Energy, contract DE-AC02-76SF00515.
} 


\section{Contents}

1 Introduction $\quad 1$

2 Effective conformal thoeries $\quad 2$

3 Three-point current correlation function $\quad 5$

$\begin{array}{lll}3.1 & \text { Review of conformal structures } & 7\end{array}$

$\begin{array}{lr}3.2 \text { Generalization to any } d>2 & 10\end{array}$

3.3 Contribution of the $F^{3}$ operator 11

$\begin{array}{lll}4 & \text { Physical measurement } & 15\end{array}$

$\begin{array}{lr}\text { A Calculation of } \mathcal{F}_{i j k}^{(3)} & 17\end{array}$

B The embedding space analogs of the conformal tensors $D_{i j k}$ and $C_{i j k} \quad 19$

\section{Introduction}

The AdS/CFT correspondence is a powerful tool for computing observables in strongly coupled systems with conformal symmetry by mapping them to weakly coupled dual gravitational theories. However, our ability to exploit the correspondence is limited by our ability to compute in the weakly coupled theory itself. For example, on the bulk AdS side, theories of practical use are not only weakly coupled, but also "well behaved," in the sense that they are effective theories describing the dynamics of only a few fields below some cutoff scale $\Lambda$. The cutoff scale suppresses non-renormalizable operators generated when fields above the cutoff scale are integrated out.

This leads to the line of enquiry: what is the class of CFTs that we can explore by mapping them to weakly coupled, well-behaved AdS duals? Put another way, what are the necessary and sufficient conditions needed for a CFT to have a weakly coupled, well behaved AdS dual?

Explorations along those lines gave rise to the idea of Effective Conformal Theories (ECT) [5]. The idea of ECTs is that the strongly coupled CFTs that can be described through weakly coupled, effective AdS bulk theories are characterized by two conditions: (1) There is a large dimension gap in the spectrum of the dilatation operator. (2) There is a small parameter that suppresses higher point connected correlation functions. The second condition is naturally satisfied in large- $N$ models where $1 / N$ plays the role of the small parameter that suppresses $n$-point connected functions as $N^{2-n}$.

In this paper we explore one consequence of such effective conformal descriptions. Assuming that such an effective description exists for a strongly coupled condensed matter 
system with non-Abelian global symmetry and dimension cutoff $\Delta$, the three-point current correlation function $\left\langle J_{i}^{a}\left(t_{1}, x\right) J_{j}^{b}\left(t_{2}, y\right) J_{k}^{c}(0)\right\rangle$ admits a perturbative expansion in the cutoff parameter $\Delta$. This parameter corresponds to $\Delta=\left(\Lambda R_{\text {AdS }}\right)$ in the effective bulk theory. The successive terms in the expansion of the three-point function can be computed from the bulk dual. In general, the successive terms are expected to carry different polarization structures $[12,13]$. The dominant contribution from the dual bulk effective AdS comes from the renormalizable (for $d \geq 4$ ) operator $\left(F_{\mu \nu}\right)^{2}$. The second contribution comes from a non-renormalizable $\left(F_{\mu \nu}\right)^{3}$ operator. In this paper we will refer to these two operators as $F^{2}$ and $F^{3}$ respectively. The latter operator is suppressed by the mass scale $\Lambda$, which gives us the boundary dimension cutoff parameter $\Delta=\Lambda R_{\mathrm{AdS}}$. Through explicit calculation, we will show that the $F^{3}$ operator indeed leads to a different polarization structure for the three-point current correlation function. This difference can be exploited to experimentally measure the expansion parameter $\Delta$ through the framework of ECTs.

The outline of the paper is as follows. An overview of ECTs is given in section 2. In section 3 , we give the derivation of the contribution of the bulk $F^{3}$ term to the boundary three-point current correlation function. The three-point functions of global non-abelian $R$-currents in strongly coupled $\mathcal{N}=4$ supersymmetric Yang-Mills theory were calculated using AdS/CFT in [8, 9]. The authors of [7] exploit the conformal symmetry constraints, in particular the inversion symmetry, to derive the dominant contribution to the threepoint current correlation function coming from $F^{2}$ term in general, non-supersymmetric CFTs dual to weakly coupled AdS. Their result for the $F^{2}$ term contribution in $d=4$ was expressed in terms of the conformal tensors $D_{i j k}(x, y, z)$ and $C_{i j k}(x, y, z)$. We give generalization of these tensor for any $d>2$ and use their technique to derive the result for the contribution of the $F^{3}$ term. The leading $F^{2}$ contribution and the first correction from the $F^{3}$ term are compared and shown to have different polarization structures. In section 4, we will outline a possible experimental measurement that can be performed to test the validity of ECTs for condensed matter systems through an indirect measurement of the cutoff dimension $\Delta$. Appendix A gives intermediate steps in the derivation of the contribution of the $F^{3}$ term. Tensor structures corresponding to $D_{i j k}$ and $C_{i j k}$ in the embedding space formalism, whose projections to the physical space reproduce $D_{i j k}$ and $C_{i j k}$, are given in appendix B.

\section{Effective conformal thoeries}

We begin with the question, "what are the necessary and sufficient conditions needed for a CFT to have a weakly coupled, well behaved AdS dual?" The necessary conditions were first motivated by locality considerations in type IIB string theory on $A d S_{5} \times S^{5} / \mathcal{N}=4$ SYM. The regime where the 10D supergravity is a good description (i.e., the regime where there is an approimate 10D flat spacetime in the neighborhood of every point) requires the mass of string excitations, of order inverse string length $l_{s}^{-1}$, to be hierarchically larger than those of the supergravity modes of order inverse AdS length $R_{\mathrm{AdS}}^{-1}$ [1]. At energies much smaller than $l_{s}^{-1}$ the theory will look like a local field theory. Since $R_{\text {AdS }}=\lambda^{1 / 4} l_{s}$, where the 't Hooft coupling $\lambda=g_{Y M}^{2} N$, the condition that $R_{\text {AdS }} \gg l_{s}$ implies that the 
't Hooft coupling must be large, $\lambda \gg 1$. Applying S-duality, which maps type IIB string theory to itself under $g_{s} \rightarrow g_{s}^{\prime}=1 / g_{s}$, and demanding that string modes remain heavy in the S-dual of the type IIB, we find another condition. Under S-duality,

$$
\begin{aligned}
& 1 \ll \lambda=g_{Y M}^{2} N \\
& \quad \stackrel{\text { s-duality } \lambda^{\prime}}{\longrightarrow}=g_{Y M}^{\prime 2} N=\frac{1}{g_{Y M}^{2}} N=\frac{N^{2}}{\lambda} .
\end{aligned}
$$

The requirement that string modes should remain heavy in both sides of the duality is the statement that both $\lambda \gg 1$ and $\lambda^{\prime} \gg 1$. We find the simultaneous requirements that $\lambda \gg 1$ and $N^{2} / \lambda \gg 1$, which are satisfied for $N^{2} \gg \lambda$, i.e, $N$ very large. But since $R_{\mathrm{AdS}} / l_{p} \sim N^{1 / 4}$, where $l_{p}$ is the Planck length, $N \gg 1$ implies that $R_{\text {AdS }} \gg l_{p}$ as well. Then we can ignore supergravity quantum corrections and consider classical or tree level supergravity.

Therefore, the gravitational bulk theory is an effective field theory with a large mass gap between the fields of mass of order $R_{\text {AdS }}^{-1}$ and high mass string and quantum gravitational excitations with masses of order $l_{s}^{-1}$ and $l_{p}^{-1}$ respectively. The effective theory has a perturbative expansion in the inverse mass gaps which suppress non-renormalizable interactions. In particular, gravitational interactions are suppressed by powers of $M_{p}^{-1}$, so we can ignore graviton exchanges.

In the dual $\mathcal{N}=4$ Super Yang-Mills theory, the large mass gap in the effective AdS translates to a large gap in operator dimensions. Further, the conformal theory has an expansion in $1 / N$, since $N$ is large. This is what mirrors the suppression by factors of $M_{p}^{-1}$ of gravitational interactions in the AdS bulk. The $1 / N$ expansion suppresses higher point connected correlation functions compared to two point functions. Based on this result, Heemskerk, Penedones, Polchiniski, and Sully [3] put forward the conjecture that any CFT with a large- $N$ like expansion and large gap in the operator dimensions has a local bulk dual AdS theory. ${ }^{1}$ The large $N$ - like expansion parameter is needed to suppress higher point connected functions compared to two point ones, which in the bulk dual corresponds to suppression of graviton mediated interactions. Fitzpatrick and Kaplan [4] have shown that with the added condition that the Mellin amplitudes of the CFT correlators have an effective theory-type expansion, we obtain the full set of necessary and sufficient conditions for a CFT to have a well behaved weakly coupled bulk AdS dual.

The picture we obtain is that the weakly coupled, well-behaved AdS duals have a double expansion in $l_{s}^{-1}$, and $l_{p}^{-1}$. The question is, what do these expansions correspond to on the CFT side? From the above paragraphs it is clear that one of these expansions is a $1 / N$ expansion which suppresses higher point connected correlation functions. But what does the expansion in the inverse dimension gap imply? Is there a concept of "Effective Conformal Theory (ECT)" that describes the dynamics of operators whose dimension lies below the cutoff dimension? If so, how does such a theory distinguish between "renormalizable" vs "non-renormalizable" interactions? What suppresses the "non-renormalizable"

\footnotetext{
${ }^{1}$ We also need all single trace operators of spin greater than two to have large dimensions since there is no known local bulk theory of particles of spin greater than 2 .
} 
operators (since conformal symmetry means that there are no mass scales)? What conditions set the range of validity for such an effective conformal theory, and where does it break down?

To address these questions, Fitzpatrick, Katz, Poland and Simmons-Duffin [5] identified these two expansions with those involving a large parameter $N$ and a large dimension gap $\Delta_{\text {gap }}=\Delta_{\text {Heavy }}-\Delta_{\text {low }}$. Such a theory is an effective conformal theory that captures the dynamics of the low-lying spectrum of the dilatation operator. Let $\Delta_{\text {low }}$ be the typical dimension of the low-lying operators, and let all other primary operators have dimension above $\Delta_{\text {Heavy }}$ which is hierarchically larger. Then there is a perturbative expansion in both $1 / \Delta_{\text {Heavy }}[6]$ and $1 / N$. The $1 / N$ suppresses all interactions, and the $1 / \Delta_{\text {Heavy }}$ suppresses higher dimensional operators in the OPE.

There is a direct parallel with effective quantum field theories. In that familiar context, there is an expansion in the small coupling constant of the effective QFT in addition to an expansion in $1 / M$, where $M$ is the scale where the effective QFT begins to break down. Analogously, in effective CFTs, the large $N$ (playing the role of the small coupling constant) ensures that connected pieces of higher point correlation functions are suppressed compared to two-point functions, whereas the small $\Delta^{-1}$ (playing the role of small $M^{-1}$ in QFTs) suppresses contributions of higher dimensional operators to the correlation function.

The schematic picture obtained is therefore the following. The dilatation operator of the CFT has a perturbative expansions in both $1 / N$ and $1 / \Delta_{\text {Heavy }}$ :

$$
D^{\mathrm{eff}}=D^{0}+\frac{1}{N}\left(V^{(1)}+\frac{1}{\Delta_{\text {Heavy }}} V^{(2)}+\ldots\right)+\mathcal{O}\left(\frac{1}{N^{2}}\right)
$$

where $D^{0}$ is the mean field dilatation operator and $V^{(1)}, V^{(2)}, \ldots$ are perturbations of the dilatation that preserve conformal symmetry.

The next question is then, "what sets the range of validity of the effective description?" The answer is again analogous to the situation in effective field theories where imposing perturbative unitarity on the Hamiltonian sets the range of validity of the effective theory. In our case, perturbative unitarity is imposed on the dilatation operator [5]. Assume $\mathcal{O}$ is the only single trace primary operator below the cutoff dimension $\Delta_{\text {Heavy. Then the }}$ low dimensional spectrum of the dilatation consists of double trace primary operators of the type $\mathcal{O}_{n, l}=\mathcal{O}\left(\partial^{2}\right)^{n}(\partial)^{l} \mathcal{O}$. These operators receive an order $1 / N$ correction to their dimension coming from the $V^{(1)}$ term; $\Delta_{n, l}=2 \Delta+2 n+l+\frac{1}{N} \gamma(n, l)$. Imposing perturbative unitarity gives a bound $|\gamma(n, l)|<4$ on the anomalous dimension $\gamma(n, l)$. However, operators $V^{(1)}$ dual to bulk interactions of mass (or scaling dimension) $\Lambda^{p}$ (hence forth refered to as "non-renormalizable" operators) lead to growth in $\gamma(n, l)$ as $n^{p-(d+1)}[3$, $5]$. Even though $\gamma(n, l)$ is an $O(1 / N)$ correction, it leads to violation of the unitarity bound for $p>d+1$ and sufficiently large $n$ no matter how small $1 / N$ may be. As $n$ approaches $\Delta_{\text {Heavy }}$, the new operators must be integrated in to moderate the growth of $\gamma(n, l)$ and restore unitarity. This will indeed be the case if the non-renormalizable operators $V$ of dimension $p$ are suppressed by $\Delta_{\text {Heavy }}^{p-(d+1)}$. In this case, $\gamma(n, l)$ grows as $\left(n / \Delta_{\text {Heavy }}\right)^{p-(d+1)}$, the unitarity bound is satisfied as long as $n<\Delta_{\text {Heavy }}$, and the ECT breaks down when $n \sim \Delta_{\text {Heavy }}$. 
This idea to use perturbative unitarity as the condition to set the range of validity of the effective description was suggested by Fitzpatrick, Katz, Poland and Simmons-Duffin in [5] as a solution to the observation made by Hofman and Maldacena in [12] that in correlation functions involving conserved currents, only certain polarization structures, those arising from the lowest dimension bulk operators appear. Demanding perturbative unitarity on all operators below the cutoff dimension $\Delta<\Delta_{\text {Heavy }}$ translates to demanding that the scale suppressing non-renormalizable operators in the bulk satisfy $\Lambda>\left(\Delta_{\text {Heavy }} / R_{\text {AdS }}\right)$. Therefore, for dimensions below $\Delta_{\text {Heavy }}$, the only polarization structures come from the lowest dimension bulk operators.

Hofman and Maldacena also observed that the number of conformally invariant structures in three-point functions of the stress tensor for $d \geq 4$ matched with the number of on-shell three-graviton scattering amplitudes in one higher dimension. This observation was generalized and proven in [13] as the statement that the number of independent tensor structures of conformally invariant three-point functions in $d$ dimensions matches exactly with the number of independent structures in scattering amplitudes of spinning particles in flat $(d+1)$-dimensional spacetime. Therefore, it is possible to argue that since $F^{2}$ and $F^{3}$ give different scattering amplitudes in $(d+1)$-dimensional flat space, they will give different polarization structures in the boundary $d$-dimensional boundary CFT as well. By explicitly computing the contribution of the bulk $F^{3}$ operator to the three-point current correlation function, we will show that this $\Delta_{\text {Heavy }}$ suppressed contribution indeed gives a polarization structure different from that of the $F^{2}$ operator. The requirement of perturbative unitarity allows us to do simple dimension counting to find the order of this suppressed contribution. We find that it is proportional to $\Delta_{\text {Heavy }}^{-2}$.

\section{Three-point current correlation function}

Armed with the above perturbative expansion, we can compute the three-point current correlation function resulting from the $F^{3}$ operator and compare the result to the contribution of the $F^{2}$ operator. It is important to note here that the system remains conformally invariant in the presence of the non-renormalizable $F^{3}$ operator. This is guaranteed by the fact that in the bulk AdS the operator is invariant under the AdS isometry. The theory we are describing thus models movement along a line of second order phase transition of a system with non-Abelian global symmetry. The coordinate along this direction is parameterized by $\Delta=\Lambda R_{\text {AdS }}$.

We begin with the bulk Euclidean action

$$
S=\frac{1}{g_{\mathrm{SG}}^{2}} \int d^{d+1} x \sqrt{g}\left(\frac{1}{2} F^{2}+\Lambda^{-p} F^{3}\right) .
$$

$g_{\mathrm{SG}}$ is the gauge coupling constant for the bulk AdS Yang-Mills theory. $\Lambda$ has mass dimension +1 . Throughout this paper we will be working in Euclidean AdS and have rescaled the gauge fields so that $A_{\mu} \rightarrow\left(i / g_{\mathrm{SG}}\right) A_{\mu}, F_{\mu \nu} \rightarrow\left(i / g_{\mathrm{SG}}\right) F_{\mu \nu}$. Further, the gauge group generators have the commutation relation $\left[T^{a}, T^{b}\right]=f^{a b c} T^{c}$. With these modifications we have

$$
F_{\mu \nu}=\partial_{\mu} A_{\nu}-\partial_{\nu} A_{\mu}+\left[A_{\mu}, A_{\nu}\right]
$$


The explicit form of the operator $F^{3}$ that we will be using is

$$
F^{3}=f^{a b c} F_{\mu \nu}^{a} F^{\nu \alpha b} F_{\alpha}^{\gamma c}
$$

However, as noted in [12] we do not need non-Abelian global symmetry to get $F^{3}$ term. If there are three $\mathrm{U}(1)$ global currents in the boundary CFT, we will get bulk interaction terms of the form $F_{\mu \alpha} F^{\alpha \nu} F_{\nu}^{\mu}$.

Dimensional analysis gives the following mass dimensions:

$$
\begin{aligned}
{\left[g_{\mathrm{SG}}\right] } & =\frac{3-d}{2} \\
{[F] } & =2 \\
p & =2 .
\end{aligned}
$$

The $F^{3}$ term gives the first sub-leading correction in the 't Hooft coupling $1 / \sqrt{\lambda}$ to the three-point current correlation function in the large $N$ limit and is unique to this order. There are no other sub-leading terms at this order in the expansion. We can prove this by writing all gauge invariant corrections in the bulk with the same mass dimension. We find the following candidate terms:

$$
\begin{aligned}
& \left(D_{\rho} F_{\mu \nu}\right)^{a}\left(D^{\rho} F^{\mu \nu}\right)^{a} \\
& \left(D_{\rho} F_{\mu \nu}\right)^{a}\left(D^{\nu} F^{\mu \rho}\right)^{a} \\
& \left(D_{\rho} D^{\rho} F_{\mu \nu}\right)^{a} F^{\mu \nu a} \\
& \left(D_{\rho} D_{\nu} F^{\rho \alpha}\right)^{a} F_{\alpha}^{\nu a} \\
& F^{3}=f^{a b c} F_{\mu \nu}^{a} F^{\nu \rho b} F_{\rho}^{\mu c} .
\end{aligned}
$$

Here, $D_{\mu}=\nabla_{\mu}+A_{\mu}$ is the coordinate and gauge covariant derivative. Other terms vanish identically by the source free equation of motion $D_{\mu} F^{\mu \nu}=0$. We can use the Bianchi identity to find a relation between the first and second terms:

$$
\left(D_{\rho} F_{\mu \nu}\right)^{a}\left(D^{\rho} F^{\mu \nu}\right)^{a}=2\left(D_{\rho} F_{\mu \nu}\right)^{a}\left(D^{\nu} F^{\mu \rho}\right)^{a}
$$

Then, integration by parts relates the first and third, as well as the second and fourth terms up to boundary terms:

$$
\begin{aligned}
& \left(D_{\rho} F_{\mu \nu}\right)^{a}\left(D^{\rho} F^{\mu \nu}\right)^{a}=-F_{\mu \nu}^{a}\left(D_{\rho} D^{\rho} F^{\mu \nu}\right)^{a}+\frac{1}{\sqrt{g}} \partial_{\rho}\left(\sqrt{g} F_{\mu \nu}^{a}\left(D^{\rho} F^{\mu \nu}\right)^{a}\right) \\
& \left(D_{\rho} F_{\mu \nu}\right)^{a}\left(D^{\nu} F^{\mu \rho}\right)^{a}=-F_{\mu \nu}^{a}\left(D_{\rho} D^{\nu} F^{\mu \rho}\right)^{a}+\frac{1}{\sqrt{g}} \partial_{\rho}\left(\sqrt{g} F_{\mu \nu}^{a}\left(D^{\rho} F^{\mu \nu}\right)^{a}\right) .
\end{aligned}
$$

The boundary terms are determined by the boundary source fields and therefore can be safely ignored in the bulk. All we have left to show is that any one of the first four terms is directly proportional to the $F^{3}$ term. We can do that by replacing $D_{\rho} D_{\nu}$ by $\left[D_{\rho}, D_{\nu}\right]+D_{\nu} D_{\rho}$ in the 4 th term in (3.3) and noting the $D_{\rho} F^{\rho \alpha}$ vanishes by the equation 
of motion.

$$
\begin{aligned}
\left(D_{\rho} D_{\nu} F^{\rho \alpha}\right)^{a} F_{\alpha}^{\nu a} & =\left(\left[D_{\rho}, D_{\nu}\right] F^{\rho \alpha}\right)^{a} F_{\alpha}^{\nu a} \\
& =R_{\nu \beta} F^{\beta \alpha a} F_{\alpha}^{\nu a}+R_{\nu \rho \beta \alpha} F^{\rho \beta a} F^{\alpha \nu a}+\frac{1}{2} f^{a b c} F_{\rho \nu}^{a} F^{\rho \alpha b} F_{\alpha}^{\nu c} \\
& =\frac{(d-1)}{R_{\mathrm{AdS}}^{2}} F_{\alpha \lambda}^{a} F^{\alpha \lambda a}+\frac{1}{2} f^{a b c} F_{\rho \nu}^{a} F^{\rho \alpha b} F_{\alpha}^{\nu c} .
\end{aligned}
$$

In the second line, use has been made of the equation $\operatorname{Tr}\left(T^{a} T^{b} T^{c}\right)=1 / 4\left(f^{a b c}-i d^{a b c}\right)$, and the fact that $d^{a b c}$ is symmetric under exchange of any two of its indices. In the last line, we've used the simple form of the curvature tensors for a maximally symmetric spacetime. In principle we could consider perturbations of the background AdS metric, but those give sub-leading corrections in $1 / N$ expansion. As we are concerned with leading order terms in $1 / N$ expansion but sub-leading in the inverse dimension expansion $\left(1 / \Delta \sim 1 / \sqrt{\lambda}=1 / \sqrt{g_{\mathrm{SG}}^{2} N}\right)$, we can ignore such perturbations of the background metric. Therefore, we conclude that $F^{3}$ is the unique sub-leading correction in the expansion in the inverse dimension.

Returning to the three-point current correlation function, we've seen that the leading $F^{2}$ and sub-leading $F^{3}$ terms fully capture all contributions to the three-point current correlation function. Let us write the action (3.1) as $S=S_{2}+S_{3}$ where $S_{2}$ is the $F^{2}$ integral and $S_{3}$ is the $F^{3}$, and proceed to study contributions to the three-point current correlation function $\left\langle J_{i}^{a}(x) J_{j}^{b}(y) J_{k}^{c}(z)\right\rangle$ coming from each of the actions $S_{2}$ and $S_{3} . i, j, k$ are $d$-dimensional Euclidean spacetime indices and $a, b, c$ label global current indices. The points $x, y, z \in \mathbb{R}^{d}$ are points in $d$-dimensional Euclidean spacetime. In this paper we adapt the notation of [7], where the contribution of $S_{2}$ has been computed. Let us first begin with a review of the conformal structures of the two and three-point current correlation functions.

\subsection{Review of conformal structures}

The two-point current correlation function in $d$-dimensions is fully determined by conformal invariance up to a normalization constant. It is given by

$$
\left\langle J_{i}^{a}(x) J_{i}^{b}(y)\right\rangle=B \delta^{a b} \frac{2(d-1)(d-2)}{(2 \pi)^{d}} \frac{J_{i j}(x-y)}{|x-y|^{2(d-1)}} .
$$

$B$ is a positive constant and

$$
J_{i j}(x)=\delta_{i j}-2 \frac{x_{i} x_{j}}{x^{2}} .
$$

The coefficient $B$ is computed from the bulk $F^{2}$ term in [7],

$$
B=\frac{1}{g_{\mathrm{SG}}^{2}} \frac{2^{d-2} \pi^{\frac{d}{2}} \Gamma(d)}{(d-1) \Gamma\left(\frac{d}{2}\right)} .
$$

The three-point current correlation function is also determined completely by conformal symmetry up to two constants. In general $d>2$ dimensions, the three-point function 
can have both parity odd and parity even components. The number of independent parity even and odd components of the three-point function of general spin $l$ primary tensors is computed using the embedding space formalism in [13], and in $(d=3)$-dimensions in [14]. For three-point functions of currents, their result gives three parity odd structures in $d=3$, and one parity odd structures in $d=4$. For $d>4$ there are no parity odd components of the three-point current correlation function. We will not discuss further the possible parity odd structures that can arise in 3 and 4 dimensions in this paper and instead focus on the parity even structures.

For three-point functions of conserved currents, the even parity three-point function in $d=4$ is given as the superposition of two permutation-odd conformal tensor structures, $D_{i j k}^{\mathrm{sym}}, C_{i j k}^{\mathrm{sym}}$ in $[10]$

$$
\left\langle J_{i}^{a}(x) J_{j}^{b}(y) J_{k}^{c}(z)\right\rangle_{+}=f^{a b c}\left(k_{1} D_{i j k}^{\mathrm{sym}}+k_{2} C_{i j k}^{\mathrm{sym}}\right)
$$

where

$$
\begin{aligned}
& D_{i j k}^{\mathrm{sym}}(x, y, z)=D_{i j k}(x, y, z)+D_{i j k}(z, x, y)+D_{i j k}(y, z, x) \\
& C_{i j k}^{\mathrm{sym}}(x, y, z)=C_{i j k}(x, y, z)+C_{i j k}(z, x, y)+C_{i j k}(y, z, x) .
\end{aligned}
$$

The tensors $D_{i j k}(x, y, z)$, and $C_{i j k}(x, y, z)$ are given by

$$
\begin{aligned}
D_{i j k}(x, y, z) & =\frac{1}{(x-y)^{2}(y-z)^{2}(z-x)^{2}} \frac{\partial}{\partial x_{i}} \frac{\partial}{\partial y_{j}} \ln \left((x-y)^{2}\right) \frac{\partial}{\partial z_{k}} \ln \left(\frac{(x-z)^{2}}{(y-z)^{2}}\right) \\
& =\frac{4}{(x-y)^{2}(y-z)^{2}(z-x)^{2}} J_{i j}(x-y) \frac{\tilde{t}_{k}}{(x-y)^{2}} \\
C_{i j k}(x, y, z) & =\frac{1}{(x-y)^{4}} \frac{\partial}{\partial x_{i}} \frac{\partial}{\partial z_{l}} \ln \left((x-z)^{2}\right) \frac{\partial}{\partial y_{j}} \frac{\partial}{\partial z_{l}} \ln \left((y-z)^{2}\right) \frac{\partial}{\partial z_{k}} \ln \left(\frac{(x-z)^{2}}{(y-z)^{2}}\right) \\
& =\frac{-8}{(x-y)^{2}(y-z)^{2}(z-x)^{2}} J_{i l}(x-z) J_{j l}(y-z) \frac{\tilde{t}_{k}}{(x-y)^{2}},
\end{aligned}
$$

where,

$$
\tilde{t}_{k}=\frac{(x-z)_{k}}{(x-z)^{2}}-\frac{(y-z)_{k}}{(y-z)^{2}}, \quad t_{k}=\frac{(y-x)_{k}}{(y-x)^{2}}-\frac{(z-x)_{k}}{(z-x)^{2}}, \quad \hat{t}_{k}=\frac{(z-y)_{k}}{(z-y)^{2}}-\frac{(x-y)_{k}}{(x-y)^{2}}
$$

The vectors $t$ and $\hat{t}$ are introduced here for later convenience since they appear in the symmetric sums of $D_{i j k}$, and $C_{i j k}$. In $d=4, C_{i j k}^{\mathrm{sym}}$ satisfies $\frac{\partial}{\partial z_{k}} C_{i j k}^{\mathrm{sym}}=0$ everywhere, whereas $D_{i j k}^{\text {sym }}$ has terms proportional to $\delta^{4}(z-x)$ and $\delta^{4}(z-y)$. Therefore, the Ward identity in $d=4$ relates the coefficient $k_{1}$ to the coefficient $B$ in (3.10) as

$$
k_{1}=\frac{B}{16 \pi^{6}} .
$$

The coefficient $k_{2}$ is undetermined. 
The contribution to the three-point function coming from the bulk action $S_{2}$ is calculated for general $d$ Euclidean spacetime dimensions in [7].

$$
\begin{aligned}
\left\langle J_{i}^{a}(x) J_{j}^{b}(y) J_{k}^{c}(z)\right\rangle^{S_{2}}= & \frac{f^{a b c}}{2 g_{\mathrm{SG}}^{2} \pi^{4}} 2\left[\mathcal{F}_{i j k}^{(2)}(x, y, z)+\mathcal{F}_{k i j}^{(2)}(z, x, y)+\mathcal{F}_{j k i}^{(2)}(y, z, x)\right], \\
\mathcal{F}_{i j k}^{(2)}(x, y, z)= & -\kappa \frac{J_{j l}(y-x)}{|y-x|^{2(d-1)}} \frac{J_{k m}(z-x)}{|z-x|^{2(d-1)}} \\
& \times \frac{1}{|t|^{d}}\left[\delta_{l m} t_{i}+(d-1) \delta_{i l} t_{m}+(d-1) \delta_{i m} t_{l}-d \frac{t_{i} t_{l} t_{m}}{|t|^{2}}\right]
\end{aligned}
$$

where,

$$
\kappa=\pi^{d / 2}\left(C^{d}\right)^{3} \frac{(d-2)}{(d-1)} \frac{\left[\Gamma\left(\frac{d}{2}\right)\right]^{3}}{[\Gamma(d)]^{2}}, \quad C^{d}=\frac{\Gamma(d)}{2 \pi^{d / 2} \Gamma\left(\frac{d}{2}\right)} .
$$

For $d=4$, the authors of [7] express the above result in terms of the conformal tensors $D_{i j k}^{\mathrm{sym}}, C_{i j k}^{\mathrm{sym}}$ elegantly as

$$
\left\langle J_{i}^{a}(x) J_{j}^{b}(y) J_{k}^{c}(z)\right\rangle^{S_{2}}=\frac{f^{a b c}}{2 g_{\mathrm{SG}}^{2} \pi^{4}}\left(D_{i j k}^{\mathrm{sym}}-\frac{1}{8} C_{i j k}^{\mathrm{sym}}\right) .
$$

Let us digress here to comment on the comparison between this bulk result for the lowest renormalizable operator $F^{2}$ in $d=4$, with the 1-loop exact two and three-point correlation function in the boundary $\mathcal{N}=4$ super-Yang-Mills theory. With the replacement $4 \pi / N \rightarrow g_{\mathrm{SG}}$ we find that both the two-point and three-point correlation functions agree exactly. From the boundary super-Yang-Mills perspective, there are no higher order corrections than the 1-loop result because of powerful non-renormalization theorems [11]. The non-renormalization for the $\mathcal{N}=4 \mathrm{SYM}$ compared to the AdS result is discussed in detail in [8], in which it was conjectured that the three-point correlation function of all chiral primary operators and their descendants is independent of $\lambda=g_{Y M}^{2} N$ to leading order in $1 / N$. On the bulk supergravity side, we would expect that gauge invariant, effective bulk operators of the form

$$
\sum_{n} \frac{1}{g_{\mathrm{SG}}^{2} \Lambda^{2 n}}\left(\left(D_{\rho} D^{\rho}\right)^{n} F_{\mu \nu} F^{\mu \nu}+D_{\mu_{1}} D_{\mu_{2}} \ldots D_{\mu_{n}} F_{\mu \nu} D^{\mu_{1}} D^{\mu_{2}} \ldots D^{\mu_{n}} F^{\mu \nu}+\ldots\right)
$$

would give order $(1 / \lambda)^{n}$ contributions to the three-point current correlation function. These are all operators of the same order in $1 / N$ expansion compared to the leading $F^{2}$ term. Through repeated use of the Bianchi identity, equations of motion, and commutations, all components of the above operators that contribute to the three-point current correlation function will have the form of $a F^{2}+b F^{3}$, where $a$ and $b$ are coefficients which are functions of $R_{\mathrm{AdS}} \Lambda \sim \sqrt{\lambda} . k_{1}$ and $k_{2}$ in the three-point function in (3.13) then in turn receive corrections proportional to $a$ and $b$ from the above non-renormalizable operators. One linear combination of $k_{1}$ and $k_{2}$ is fixed by the Ward identity which relates the three-point current correlation function with the two-point current correlation function, but the orthogonal linear combination is undetermined. The conjectured independence of the three-point 
correlation function on $\lambda$ in [8] in the case of $\mathcal{N}=4 \mathrm{SYM}$ then requires that all dependence of $k_{1}$ and $k_{2}$ on $a$ and $b$ must cancel, i.e., all contributions from (3.24) cancel. This cancelation can not result from conformal symmetry since, as we noted at the beginning of this section, all of these operators give conformally invariant correlation functions in the boundary (because they are invariant under the bulk AdS inversion isometry). Therefore, the cancelation must be the result of the $\mathcal{N}=1$ supergravity of the $A d S_{5} \times S^{5}$.

If we remove supersymmetry from both sides of the duality, non-renormalizable operators of the form (3.24) will give $k_{1}$ and $k_{2}$ dependence on $a$ and $b$. The claim of effective conformal theory [5] is that computing the corrections from (3.24) using Witten diagrams in the bulk is the same as computing the expansion of the three-point current correlation function in $1 / \Delta$ with successive terms coming from conformally invariant, non-renormalizable perturbations of the dilatation operator (corresponding to the bulk operators (3.24)) suppressed by powers of dimension cutoff $\Delta$ as prescribed by perturbative unitarity.

\subsection{Generalization to any $d>2$}

When generalizing (3.23) to any $d>2$ dimensions, we first note that the symmetric tensor $J_{i j}$ which appears in the two-point function in (3.10) remains unchanged since it comes from general requirements of covariance under the conformal algebra [15]. The tensors $D_{i j k}(x, y, z)$, and $C_{i j k}(x, y, z)$ can be generalized as follows.

$$
\begin{aligned}
D_{i j k}(x, y, z) & =\frac{1}{(|x-y||y-z||z-x|)^{d-2}} \frac{\partial}{\partial x_{i}} \frac{\partial}{\partial y_{j}} \ln \left(|x-y|^{d-2}\right) \frac{\partial}{\partial z_{k}} \ln \left(\frac{|x-z|^{d-2}}{|y-z|^{d-2}}\right) \\
& =\frac{(d-2)^{2}}{(|x-y||y-z||z-x|)^{d-2}} J_{i j}(x-y) \frac{\tilde{t}_{k}}{|x-y|^{2}} \\
C_{i j k}(x, y, z) & =\frac{1}{|x-y|^{d}} \frac{\partial}{\partial x_{i}} \frac{\partial}{\partial z_{l}} \ln \left(|x-z|^{d-2}\right) \frac{\partial}{\partial y_{j}} \frac{\partial}{\partial z_{l}} \ln \left(|y-z|^{d-2}\right) \frac{\partial}{\partial z_{k}} \ln \left(\frac{|x-z|^{d-2}}{|y-z|^{d-2}}\right) \\
& =\frac{-(d-2)^{3}}{(|x-y||y-z||z-x|)^{d-2}} J_{i l}(x-z) J_{j l}(y-z) \frac{\tilde{t}_{k}}{|x-y|^{2}} .
\end{aligned}
$$

The symmetric sums of the tensors, $D_{i j k}^{\mathrm{sym}}, C_{i j k}^{\mathrm{sym}}$ have the following property:

$$
\begin{aligned}
\frac{\partial}{\partial z_{k}} D_{i j k}^{\mathrm{sym}} & =(d-2)^{2} S_{d}\left(\frac{d+2}{d}\right) \frac{J_{i j}(x-y)}{|x-y|^{2(d-1)}}\left(\delta^{d}(z-y)-\delta^{d}(z-x)\right) \\
\frac{\partial}{\partial z_{k}} C_{i j k}^{\mathrm{sym}} & =-(d-2)^{3} S_{d}\left(\frac{d-4}{d}\right) \frac{J_{i j}(x-y)}{|x-y|^{2(d-1)}}\left(\delta^{d}(z-y)-\delta^{d}(z-x)\right),
\end{aligned}
$$

where,

$$
S_{d}=\frac{2 \pi^{\frac{d}{2}}}{\Gamma\left(\frac{d}{2}\right)} .
$$

We have used the following formulae to derive the above result:

$$
\lim _{x \rightarrow 0} \frac{x_{i} x_{j}}{x^{2}}=\frac{1}{d} \delta_{i j}, \quad \lim _{z \rightarrow x} \frac{\partial}{\partial z_{k}}\left(\frac{(z-x)_{k}}{|z-x|^{d}}\right)=S_{d} \delta^{d}(z-x)
$$


The Ward identity in $d$-dimensions relates one linear combination of $k_{1}$ and $k_{2}$ to $B$.

$$
B=\frac{(2 \pi)^{d} S_{d}}{2} \frac{(d-2)}{(d-1)}\left(\frac{(d+2)}{d} k_{1}-\frac{(d-2)(d-4)}{d} k_{2}\right)
$$

In $d=4$ we recover $(3.21)$.

It will be interesting to find the analogues of the tensor structures $C_{i j k}, D_{i j k}$ in the embedding space formalism used in [13] and [16]. This is done in appendix A.

To compare the contribution of the $F^{3}$ operator to the three-point function with that coming from the $F^{2}$ operator in general $d>2$ dimensions, it is helpful to find an expression to (3.22) analogous to (3.23) for general $d>2$. This can be achieved using the formulae

$$
\begin{aligned}
J_{k m}(z-x) t_{m} & =-\frac{(y-z)^{2}}{(y-x)^{2}} \tilde{t}_{k}, \text { and } \\
J_{j l}(y-x) t_{l} & =-\frac{(z-y)^{2}}{(z-x)^{2}} \hat{t} .
\end{aligned}
$$

We then find

$$
\left\langle J_{i}^{a}(x) J_{j}^{b}(y) J_{k}^{c}(z)\right\rangle^{S_{2}}=\frac{f^{a b c} \kappa}{2 g_{\mathrm{SG}}^{2}} \frac{(3 d-4)}{(d-2)^{2}}\left(D_{i j k}^{\mathrm{sym}}-\frac{1}{(3 d-4)} C_{i j k}^{\mathrm{sym}}\right) .
$$

\subsection{Contribution of the $F^{3}$ operator}

From the AdS/CFT ansatz for correlation functions [2], we have

$$
\left\langle\exp \int J_{i}^{a} A_{0}^{a i}\right\rangle_{\mathrm{CFT}}=Z_{S}\left(A_{0}\right)
$$

where $Z_{S}\left(A_{0}\right)$ is the bulk path integral for the gauge field $A\left(x_{0}, x\right)$ expressed in terms of the boundary value $A_{0}(x)$. In the limit where the bulk gravitational theory is weakly coupled, the path integral is approximately the classical path integral,

$$
Z_{S}\left(A_{0}\right) \simeq \exp \left(-I_{s}\left(A_{0}\right)\right)
$$

where $I_{s}\left(A_{0}\right)$ is the action expressed in terms of the boundary value of the field $A$ at boundary coordinates, $x, y, z$. In the following, Latin indices $i, j, k$ run from 1 to $d$, and Greek letters $\mu, \nu$ run from 0 to $d$, where 0 is the extra AdS coordinate.

We are interested in the connected three-point correlator,

$$
\begin{aligned}
\left\langle J_{i}^{a}(x) J_{j}^{b}(y) J_{k}^{c}(z)\right\rangle_{\text {connected }} & =\frac{\delta}{\delta A_{0}^{a i}(x)} \frac{\delta}{\delta A_{0}^{b j}(y)} \frac{\delta}{\delta A_{0}^{c k}(z)} \log \left(Z_{S}\left(A_{0}\right)\right) \\
& =\left.\frac{\delta}{\delta A_{0}^{a i}(x)} \frac{\delta}{\delta A_{0}^{b j}(y)} \frac{\delta}{\delta A_{0}^{c k}(z)}\left(-I_{s}\left(A_{0}\right)\right)\right|_{A_{0}=0}
\end{aligned}
$$

To compute the contribution of the $F^{3}$ operator, we begin by expressing the $S_{3}$ part of the action in terms of the boundary value of the gauge field and the boundary-to-bulk 
Green's function $G_{\mu i}^{a b}\left(w_{0}, x ; 0, \tilde{x}\right)$, where $x, \tilde{x}$ are the $d$-dimensional boundary coordinates and $w_{0}$ is the perpendicular bulk coordinate.

$$
\begin{aligned}
& A_{\mu}^{a}\left(w_{0}, \tilde{x}\right)=\int d^{d} x G_{\mu i}^{a b}\left(w_{0}, \tilde{x} ; 0, x\right) A_{0}^{i b}(0, x), \quad \text { where } \quad G_{\mu i}^{a b}=G_{\mu i} \delta^{a b} \text { and so } \\
& A_{\mu}^{a}\left(w_{0}, \tilde{x}\right)=\int d^{d} x G_{\mu i}\left(w_{0}, \tilde{x} ; 0, x\right) A_{0}^{i a}(0, x) .
\end{aligned}
$$

Plugging this into the $S_{3}$ part of the bulk action in (3.1) and evaluating (3.35) we find the following expression.

$$
\begin{aligned}
\left\langle J_{i}^{a}(x) J_{j}^{b}(y) J_{k}^{c}(z)\right\rangle_{\text {connected }}^{S_{3}} & =\left.\frac{\delta}{\delta A_{0}^{a i}(x)} \frac{\delta}{\delta A_{0}^{b j}(y)} \frac{\delta}{\delta A_{0}^{c k}(z)}\left(-S_{3}\right)\right|_{A_{0}=0} \\
& =\frac{1}{\Lambda^{p} g_{\mathrm{SG}}^{2}} 2 f^{a b c}\left[\mathcal{F}_{i j k}^{(3)}+\mathcal{F}_{j k i}^{(3)}+\mathcal{F}_{k i j}^{(3)}\right]
\end{aligned}
$$

where

$$
\mathcal{F}_{i j k}^{(3)}=\int d^{d+1} w \sqrt{g} \partial_{[\mu} G_{\alpha] i}(w, x) \partial_{[\nu} G_{\beta] j}(w, y) \partial_{[\rho} G_{\gamma] k}(w, z) g^{\alpha \nu} g^{\beta \rho} g^{\gamma \mu}
$$

We evaluate $\mathcal{F}_{i j k}^{(3)}$ in Euclidean AdS, in the parameterization of AdS as the Lobachevsky upper half space with the metric

$$
d s^{2}=\frac{R_{\mathrm{AdS}}^{2}}{w_{0}^{2}}\left(d w_{0}^{2}+\sum_{\mu=1}^{d} d x_{\mu}^{2}\right) .
$$

We set $R_{\text {AdS }}=1$ in the following computation and restore it in the final answer by dimensional analysis.

The boundary-to-bulk propagator of the gauge field from the boundary point $x^{\mu}=$ $(0, x)^{\mu}$ to the bulk point $w^{\mu}=\left(w_{0}, \tilde{x}\right)^{\mu}$ is given explicitly in [7]

$$
G_{\mu i}\left(w_{0}, \tilde{x} ; 0, x\right)=C^{d} \frac{w_{0}^{d-2}}{\left[w_{0}^{2}+(\tilde{x}-x)^{2}\right]^{d-1}} J_{\mu i}(w-x) .
$$

We will use the technique described by Freedman, Mathur, Matusis, and Rastelli [7] to evaluate $\mathcal{F}_{i j k}^{(3)}$. Their technique takes advantage of the fact that the Green's function has translation invariance in the boundary coordinates.

$$
\left\langle J_{i}^{a}(x) J_{j}^{b}(y) J_{k}^{c}(z)\right\rangle=\left\langle J_{i}^{a}(0) J_{j}^{b}(y-x) J_{k}^{c}(z-x)\right\rangle
$$

Evaluating $\langle J(0) J(y-x) J(z-x)\rangle$ is easier because there are only two terms in the denominator of (3.38). We begin by calculating $\left\langle J_{i}^{a}(0) J_{j}^{b}(y) J_{k}^{c}(z)\right\rangle$. Using the metric (3.39) in the formula for $\mathcal{F}_{i j k}$ we find,

$$
\mathcal{F}_{i j k}^{(3)}=\int d^{d} x^{\prime} d w_{0} \frac{w_{0}^{6}}{w_{0}^{d+1}} \partial_{[\mu} G_{\nu] i}\left(x^{\prime}, 0\right) \partial_{[\nu} G_{\rho] j}\left(x^{\prime}, y\right) \partial_{[\rho} G_{\mu] k}\left(x^{\prime}, z\right) .
$$


To simplify the above integral further we will take advantage of the inversion isometry of the AdS metric. The transformation

$$
w_{0}=\frac{w_{0}^{\prime}}{w_{0}^{\prime 2}+x^{\prime 2}}, \quad x^{\mu}=\frac{x^{\prime \mu}}{w_{0}^{\prime 2}+x^{\prime 2}}
$$

on the AdS coordinates leaves the metric (3.39) invariant. On the other hand, such a transformation acts as conformal isometry on the boundary coordinates; the flat boundary metric $d s^{2}=\sum_{i} d x^{i} d x^{i} \rightarrow \frac{1}{|x|^{4}} \sum_{i} d x^{i} d x^{i}$ under

$$
x^{i}=\frac{x^{i}}{x^{\prime 2}}
$$

The Jacobian of the inversion transformation inherits the tensor structure of $J_{\mu \nu}$

$$
\begin{aligned}
\frac{\partial w_{\mu}^{\prime}}{\partial w_{\nu}} & =w^{\prime 2}\left(\delta_{\mu \nu}-2 \frac{w_{\mu}^{\prime} w_{\nu}^{\prime}}{w^{\prime 2}}\right) \\
& =w^{\prime 2} J_{\mu \nu}\left(w^{\prime}\right)=\frac{1}{w^{2}} J_{\mu \nu}(w)
\end{aligned}
$$

$J_{\mu \nu}$ satisfies the following identities:

$$
\begin{aligned}
J_{\mu \nu}(w-u) & =J_{\mu \rho}\left(w^{\prime}\right) J_{\rho \sigma}\left(w^{\prime}-u^{\prime}\right) J_{\sigma \nu}\left(u^{\prime}\right) \\
J_{\mu \nu}(w) J_{\nu \rho}(w) & =\delta_{\mu \rho} .
\end{aligned}
$$

Using these identities and explicit formula for $G_{\mu \nu}$ we can show that it transforms as a covariant rank 2 tensor with scaling dimension $d-2$ under the simultaneous bulk and boundary inversions.

$$
\begin{aligned}
G_{\mu i}\left(w_{0}, \tilde{x} ; 0, x\right) & =C^{d} \frac{1}{w_{0}}\left(\frac{w_{0}}{w_{0}^{2}+(\tilde{x}-x)^{2}}\right)^{d-1} J_{\mu i}(w-x) \\
& =C^{d} \frac{w^{\prime 2}}{w_{0}^{\prime}}\left(\frac{w_{0}^{\prime}}{w_{0}^{\prime 2}+\left(\tilde{x}^{\prime}-x^{\prime}\right)^{2}}\right)^{d-1}\left|x^{\prime}\right|^{2(d-1)} J_{\mu \rho}\left(w^{\prime}\right) J_{\rho k}\left(w^{\prime}-x^{\prime}\right) J_{k i}\left(x^{\prime}\right) \\
& =w^{\prime 2} J_{\mu \rho}\left(w^{\prime}\right)\left|x^{\prime}\right|^{2} J_{k i}\left(x^{\prime}\right)\left|x^{\prime}\right|^{2(d-2)} G_{\mu i}\left(w^{\prime}, x^{\prime}\right) \\
& =\frac{\partial w_{\nu}^{\prime}}{\partial w_{\mu}} \frac{\partial x_{k}^{\prime}}{\partial x_{i}}\left|x^{\prime}\right|^{2(d-2)} G_{\nu k}\left(w^{\prime}, x^{\prime}\right) \\
& =\frac{\partial w_{\nu}^{\prime}}{\partial w_{\mu}} \frac{\partial x_{k}^{\prime}}{\partial x_{i}} G_{\nu k}^{\prime}\left(w^{\prime}, x^{\prime}\right) .
\end{aligned}
$$

In the second line, $w^{\prime \mu}=\left(w_{0}^{\prime}, \tilde{x}^{\prime}\right)^{\mu}$. Similarly, $\partial_{[\mu} G_{\nu] i}(w, x)$ transforms covariantly as

$$
\begin{aligned}
\partial_{[\mu} G_{\nu] i}(w, x) & =w^{\prime 2} J_{\mu \alpha}\left(w^{\prime}\right) w^{\prime 2} J_{\nu \beta}\left(w^{\prime}\right)\left|x^{\prime}\right|^{2} J_{i k}\left(x^{\prime}\right)\left|x^{\prime}\right|^{2(d-2)} \partial_{[\alpha}^{\prime} G_{\beta] k}\left(w^{\prime}, x^{\prime}\right) \text {, where } \\
\partial^{\prime} & =\frac{\partial}{\partial w^{\prime}} .
\end{aligned}
$$

When we set $x$ to zero and do an inversion transformation, we find the following simpler forms

$$
\begin{aligned}
G_{\mu i}(w, 0) & =C^{d}\left(w_{0}^{\prime}\right)^{d-2} w^{\prime 2} J_{\mu i}\left(w^{\prime}\right) \\
\partial_{[\mu} G_{\nu] i}(w, 0) & =(d-2) C^{d}\left(w_{0}^{\prime}\right)^{d-3}\left(w^{\prime}\right)^{4} J_{0[\mu}\left(w^{\prime}\right) J_{\nu] i}\left(w^{\prime}\right) .
\end{aligned}
$$


Applying the inversion on (3.41) and simplifying, we find

$$
\begin{aligned}
\mathcal{F}_{i j k}^{(3)}(0, y, z)=(d-2)^{3}\left(C^{d}\right)^{3}|y|^{2(d-1)} J_{a j}\left(y^{\prime}\right)|z|^{2(d-1)} J_{b k}(z) & \int d^{d} w^{\prime} d w_{0}^{\prime} \frac{\left(w_{0}^{\prime}\right)^{2 d-4}}{\left[w_{0}^{\prime 2}+\left(\tilde{x}^{\prime}-y^{\prime}\right)^{2}\right]^{d-1}\left[w_{0}^{\prime 2}+\left(\tilde{x}^{\prime}-z^{\prime}\right)^{2}\right]^{d-1}} \\
& \left(J_{0[i}\left(w^{\prime}-y^{\prime}\right) J_{\gamma] a}\left(w^{\prime}-y^{\prime}\right) J_{0[\gamma}\left(w^{\prime}-z^{\prime}\right) J_{0] b}\left(w^{\prime}-z^{\prime}\right)\right. \\
& \left.+J_{0[\gamma}\left(w^{\prime}-y^{\prime}\right) J_{0] a}\left(w^{\prime}-y^{\prime}\right) J_{0[\gamma}\left(w^{\prime}-z^{\prime}\right) J_{i] b}\left(w^{\prime}-z^{\prime}\right)\right) .
\end{aligned}
$$

After performing the integral and expressing the result in terms of the tensors $D_{i j k}, C_{i j k}$, we find the following simple form:

$$
\mathcal{F}_{i j k}^{(3)}(x, y, z)=-\frac{\kappa d}{2}\left(D_{j k i}(y, z, x)+\frac{1}{d} C_{j k i}(y, z, x)\right) .
$$

The intermediate steps are included in appendix B. The symmetric sum then becomes

$$
\mathcal{F}_{i j k}^{(3) \operatorname{sym}}(x, y, z)=-\frac{\kappa d}{2}\left(D_{i j k}^{\mathrm{sym}}(y, z, x)+\frac{1}{d} C_{i j k}^{\mathrm{sym}}(y, z, x)\right) .
$$

For comparison, the contribution of the $F^{2}$ operator to three-point current correlation function, given in $(3.22)$ is

$$
\mathcal{F}_{i j k}^{(2) \mathrm{sym}}=\frac{\kappa(3 d-4)}{2(d-2)^{2}}\left(D_{i j k}^{\mathrm{sym}}(x, y, z)-\frac{1}{(3 d-4)} C_{i j k}^{\mathrm{sym}}(x, y, z)\right) .
$$

As expected, the polarization structure resulting from the $F^{3}$ operator is different from the $F^{2}$ contribution.

After restoring the correct factor of $R_{\text {AdS }}$ by dimensional analysis, and letting $R_{\text {AdS }} \Lambda=$ $\Delta$, the three-point current contributions of each of the operators $F^{2}$ and $F^{3}$ are

$$
\begin{aligned}
&\left\langle J_{i}^{a}(x) J_{j}^{b}(y) J_{k}^{c}(z)\right\rangle^{S_{2}}=f^{a b c} \kappa\left(\frac{\left(R_{\mathrm{AdS}}\right)^{d-3}}{g_{\mathrm{SG}}^{2}}\right)\left(\frac{(3 d-4)}{2(d-2)^{2}}\right) \\
&\left(D_{i j k}^{\mathrm{sym}}(x, y, z)-\frac{1}{(3 d-4)} C_{i j k}^{\mathrm{sym}}(x, y, z)\right) \\
&\left\langle J_{i}^{a}(x) J_{j}^{b}(y) J_{k}^{c}(z)\right\rangle^{S_{3}=}=-f^{a b c} \kappa\left(\frac{\left(R_{\mathrm{AdS}}\right)^{d-3}}{\Delta^{2} g_{\mathrm{SG}}^{2}}\right) d\left(D_{i j k}^{\mathrm{sym}}(y, z, x)+\frac{1}{d} C_{i j k}^{\mathrm{sym}}(y, z, x)\right) .
\end{aligned}
$$

The sum of the two contributions is,

$$
\begin{aligned}
\left\langle J_{i}^{a}(x) J_{j}^{b}(y) J_{k}^{c}(z)\right\rangle^{S_{2}+S_{3}}= & f^{a b c} \kappa\left(\frac{\left(R_{\mathrm{AdS}}\right)^{d-3}}{g_{\mathrm{SG}}^{2}}\right)\left(\frac{3 d-4}{2(d-2)^{2}}\right) \\
& {\left[\left(1-\frac{2 d(d-2)^{2}}{(3 d-4) \Delta^{2}}\right) D_{i j k}^{\mathrm{sym}}-\frac{1}{3 d-4}\left(1+\frac{2(d-2)^{2}}{\Delta^{2}}\right) C_{i j k}^{\mathrm{sym}}\right] . }
\end{aligned}
$$


In particular, for $d=3$,

$$
\begin{aligned}
\mathcal{F}_{i j k}^{(3) \mathrm{sym}} & =-\frac{1}{2^{9}}\left(D_{i j k}^{\mathrm{sym}}+\frac{1}{3} C_{i j k}^{\mathrm{sym}}\right) \\
\mathcal{F}_{i j k}^{(2) \mathrm{sym}} & =\frac{5}{2^{10}}\left(D_{i j k}^{\mathrm{sym}}-\frac{1}{5} C_{i j k}^{\mathrm{sym}}\right) \\
\left\langle J_{i}^{a}(x) J_{j}^{b}(y) J_{k}^{c}(z)\right\rangle & =f^{a b c}\left(\frac{5}{2^{10} g_{\mathrm{SG}}^{2}}\right)\left(\left(1-\frac{6}{5 \Delta^{2}}\right) D_{i j k}^{\mathrm{sym}}-\frac{1}{5}\left(1+\frac{2}{\Delta^{2}}\right) C_{i j k}^{\mathrm{sym}}\right) .
\end{aligned}
$$

In $d=4$, the combined three-point current correlation function is

$$
\begin{aligned}
\mathcal{F}_{i j k}^{(3) \mathrm{sym}} & =-\frac{1}{\pi^{4}}\left(D_{i j k}^{\mathrm{sym}}+\frac{1}{4} C_{i j k}^{\mathrm{sym}}\right) \\
\mathcal{F}_{i j k}^{(2) \mathrm{sym}} & =\frac{1}{2 \pi^{4}}\left(D_{i j k}^{\mathrm{sym}}-\frac{1}{8} C_{i j k}^{\mathrm{sym}}\right) \\
\left\langle J_{i}^{a}(x) J_{j}^{b}(y) J_{k}^{c}(z)\right\rangle & =f^{a b c}\left(\frac{R_{\mathrm{AdS}}}{2 \pi^{4} g_{\mathrm{SG}}^{2}}\right)\left(\left(1-\frac{1}{4 \Delta^{2}}\right) D_{i j k}^{\mathrm{sym}}-\frac{1}{8}\left(1+\frac{8}{\Delta^{2}}\right) C_{i j k}^{\mathrm{sym}}\right) .
\end{aligned}
$$

These results are the two lowest order results to the three-point current correlation function in the $1 / \Delta$ expansion and leading order in $1 / N$ expansion. The first $\mathcal{O}\left(1 / \Delta^{2}\right)$ correction to the three-point current correlation function comes entirely from the nonrenormalizable $F^{3}$ operator.

\section{Physical measurement}

Measuring the three-point spin-current in condensed matter systems directly is near impossible through existing technologies. However, measurements that look for non-linear Ohm's-law type effects in induced spin-currents contain data about the three-point current correlation function. In the presence of an external field $\vec{E}$ the induced current will take the form,

$$
J_{k}^{c}=\sigma_{i k}^{a c} E_{a}^{i}+\rho_{i j k}^{a b c} E_{a}^{i} E_{b}^{j}+\mathcal{O}\left(E^{3}\right),
$$

With $a, b, c$ indices of global currents, and $i, j, k$ indices of $d$-dimensional Euclidean spacetime coordinates. $\sigma_{i j}^{a b}$ and $\rho_{i j k}^{a b c}$ are the 2 and 3-rank conductivity tensors. The fact that the two operators lead to different polarization structures will be exploited. Consider the special points

$$
\begin{aligned}
& z=(0,0,0, \ldots, 0) \\
& x=(\tau, r, 0, \ldots, 0) \\
& y=(\tau,-r, 0, \ldots, 0) .
\end{aligned}
$$

The $i=j=k$ component of the tensor $D_{i j k}^{\mathrm{sym}}$ automatically vanishes, whereas the $i j k=122$ component of $D_{122}^{\mathrm{sym}}$ is just a rescaling of $C_{i j k}^{\mathrm{sym}}$. However, the $i j k=112$ component of the 
symmetric tensors $D_{112}^{\mathrm{sym}}$ and $C_{112}^{\mathrm{sym}}$ are linearly independent, and take the values

$$
\begin{aligned}
& D_{112}^{\mathrm{sym}}=\frac{(d-2)^{2}}{\left[2 r\left(\tau^{2}+r^{2}\right)\right]^{(d-1)}}\left(\frac{r^{4}-\tau^{4}+8 \tau^{2} r^{2}}{\left(\tau^{2}+r^{2}\right)^{2}}\right), \\
& C_{112}^{\mathrm{sym}}=-\frac{(d-2)^{3}}{\left[2 r\left(\tau^{2}+r^{2}\right)\right]^{(d-1)}}\left(1-\frac{16 \tau^{2} r^{2}}{\left(\tau^{2}+r^{2}\right)^{2}}\right) .
\end{aligned}
$$

Then, the two different linear combinations corresponding to the contribution of the $F^{2}$ operator verses the $F^{3}$ operator vanish for different values of $\tau$ and $r$. For example, for $d=3$ Euclidean dimensions,

$$
\left\langle J_{1}^{a}(x) J_{1}^{b}(y) J_{2}^{c}(z)\right\rangle=\frac{f^{a b c}}{2^{9} g_{\mathrm{SG}}^{2}} \frac{1}{\left[2 r\left(\tau^{2}+r^{2}\right)\right]^{4}}\left(\left(3 r^{4}-2 \tau^{4}+13 \tau^{2} r^{2}\right)-\frac{2}{\Delta^{2}}\left(r^{4}-2 \tau^{4}+19 \tau^{2} r^{2}\right)\right) .
$$

Comparing two measurements at two different set of points where either contribution vanishes, we can not only test the validity of the effective approach, but also find the dimension gap $\Delta$ suppressing higher order corrections.

To conclude, in this paper we computed the three-point current correlation function in the framework of Effective Conformal Field Theory. This describes the dynamics of all operators with dimensions below the cutoff dimension $\Delta_{\text {Heavy }}$. In systems with large dimension gap $\Delta_{\text {gap }} \approx \Delta_{\text {heavy }}$ and a $1 / N$ like suppression, there is double expansion in both $1 / N$ and $1 / \Delta_{\text {gap }}$. The contributions to the three-point current correlation function coming from the lowest non-renormalizable bulk operator $F^{3}$ is computed and compared to the contribution coming from the renormalizable $F^{2}$ bulk operator already computed in the literature. It is shown that the two operators give rise to different polarization structure of the three-point current correlation function. The polarization structure coming from the non-renormalizable bulk $F^{3}$ term is suppressed by powers of the cutoff dimension $\Delta_{\text {Heavy }}$ prescribed by demanding perturbative unitarity.

By measuring the non-linear response to external fields, it is possible to test the effective description for strongly coupled condensed matter systems. In systems with global non-Abelian symmetry and large hierarchy in operator dimensions at second order phase transition, we can expect new terms of order $1 / \Delta_{\text {Heavy }}^{2}$ in the three-point current correlation function with a different polarization structure to the leading effect.

\section{Acknowledgments}

This humble effort would not have been possible without the help of persons whom I would like to acknowledge in this section. First and foremost I thank God to whom this work, the first fruit of my labor in graduate school, is dedicated. I thank Jared Kaplan for suggesting the project and for many helpful discussions, both physics related and otherwise. I thank Michael Peskin for being very supportive of my efforts. I also thank Srinivas Raghu and Maissam Barkeshli for helpful conversations about the experimental aspects of the work, and Laim Fitzpatrick for helpful feedback. The work was supported by DOE under contract DE-AC02-76SF00515. 


\section{A Calculation of $\mathcal{F}_{i j k}^{(3)}$}

We begin with (3.52).

$$
\begin{aligned}
\mathcal{F}_{i j k}^{(3)}(0, y, z)=(d-2)^{3}\left(C^{d}\right)^{3}|y|^{2(d-1)} J_{a j}\left(y^{\prime}\right)|z|^{2(d-1)} J_{b k}(z) & \int d^{d} w^{\prime} d w_{0}^{\prime} \frac{\left(w_{0}^{\prime}\right)^{2 d-4}}{\left[w_{0}^{\prime 2}+\left(\tilde{x}^{\prime}-y^{\prime}\right)^{2}\right]^{d-1}\left[w_{0}^{\prime 2}+\left(\tilde{x}^{\prime}-z^{\prime}\right)^{2}\right]^{d-1}} \\
& \left(J_{0[i}\left(w^{\prime}-y^{\prime}\right) J_{\gamma] a}\left(w^{\prime}-y^{\prime}\right) J_{0[\gamma}\left(w^{\prime}-z^{\prime}\right) J_{0] b}\left(w^{\prime}-z^{\prime}\right)\right. \\
& \left.+J_{0[\gamma}\left(w^{\prime}-y^{\prime}\right) J_{0] a}\left(w^{\prime}-y^{\prime}\right) J_{0[\gamma}\left(w^{\prime}-z^{\prime}\right) J_{i] b}\left(w^{\prime}-z^{\prime}\right)\right) .
\end{aligned}
$$

The following integral appears repeatedly in the evaluation of $\mathcal{F}_{i j k}^{(3)}$. It was performed using Feynman parameters in [7]. In the following $x, y, z, w$ are coordinates in the $d$-dimensional boundary, and $z_{0}, w_{0}$ are perpendicular bulk coordinates.

$$
\begin{aligned}
\int_{0}^{\infty} d z_{0} \int d^{d} z \frac{z_{0}^{a}}{\left[z_{0}^{2}+(z-x)^{2}\right]^{b}\left[z_{0}^{2}+(z-y)^{2}\right]^{c}} \equiv & I[a, b, c, d]|x-y|^{1+a+d-2 b-2 c} \\
I[a, b, c, d]= & \frac{\pi^{\frac{d}{2}} \frac{\Gamma\left(\frac{a+1}{2}\right) \Gamma\left(b+c-\frac{d}{2}-\frac{a+1}{2}\right)}{2}}{\Gamma(b) \Gamma(c)} \\
& \frac{\Gamma\left(\frac{a+1}{2}+\frac{d}{2}-b\right) \Gamma\left(\frac{a+1}{2}+\frac{d}{2}-c\right)}{\Gamma(a+1+d-b-c)} .
\end{aligned}
$$

We can proceed in the evaluation of $\mathcal{F}_{i j k}^{(3)}$ by expressing the tensors in the integrand in terms of derivatives of the integrand in the left hand side of (A.2) as follows:

$$
\begin{aligned}
\frac{J_{k l}(w-t)}{\left[w_{0}^{2}+(w-t)^{2}\right]^{d-1}}= & \left(\frac{d}{d-1}\right) \frac{\delta_{k l}}{\left[w_{0}^{2}+(w-t)^{2}\right]^{d-1}} \\
& -\frac{1}{2(d-1)(d-2)} \frac{\partial}{\partial t_{k}} \frac{\partial}{\partial t_{l}}\left(\frac{1}{\left[w_{0}^{2}+(w-t)^{2}\right]^{d-2}}\right) \\
\frac{(w-t)_{j}(w-t)_{i}}{\left[w_{0}^{2}+(w-t)^{2}\right]^{d}}= & \frac{1}{2(d-1)} \frac{\delta_{j i}}{\left[w_{0}^{2}+(w-t)^{2}\right]^{d-1}} \\
& +\frac{1}{4(d-2)(d-1)} \frac{\partial}{\partial t_{j}} \frac{\partial}{\partial t_{i}}\left(\frac{1}{\left[w_{0}^{2}+(w-t)^{2}\right]^{d-2}}\right) \\
\frac{J_{i[j}(w-t) J_{k] l}(w-t)}{\left[w_{0}^{2}+(w-t)^{2}\right]^{d-1}}= & \frac{\delta_{i[j} \delta_{k] l}}{\left[w_{0}^{2}+(w-t)^{2}\right]^{d-1}} \\
& -\frac{1}{2(d-1)(d-2)}\left(\delta_{i[j} \partial_{k]}^{t} \partial_{l}^{t}+\delta_{l[k} \partial_{j]}^{t} \partial_{i}^{t}\right)\left(\frac{1}{\left[w_{0}^{2}+(w-t)^{2}\right]^{d-1}}\right)
\end{aligned}
$$

where $t$ is given in (3.20), and

$$
\partial_{k}^{t}=\frac{\partial}{\partial t_{k}}
$$


The integral on the right hand side of eq. (3.52) now simplifies to the following.

$$
\begin{gathered}
\int d^{d} w^{\prime} d w_{0}^{\prime}\left(w_{0}^{\prime}\right)^{2 d-4}\left(A+B+C-A^{\prime}-B^{\prime}-C^{\prime}\right) \\
A=-\frac{2}{d-1} \delta_{a[b} \partial_{i]}^{y^{\prime}} \frac{w_{0}^{\prime 3}}{\left[w_{0}^{\prime 2}+\left(\overrightarrow{w^{\prime}}-y^{\prime}\right)^{2}\right]^{d-1}\left[w_{0}^{\prime 2}+\left(\overrightarrow{w^{\prime}}-z^{\prime}\right)^{2}\right]^{d}} \\
B=-\frac{1}{d-1} \delta_{a[i} \partial_{b]}^{y^{\prime}} \frac{w_{0}^{\prime}}{\left[w_{0}^{\prime 2}+\left(\overrightarrow{w^{\prime}}-y^{\prime}\right)^{2}\right]^{d-1}\left[w_{0}^{\prime 2}+\left(\overrightarrow{w^{\prime}}-z^{\prime}\right)^{2}\right]^{d-1}} \\
C=-\frac{1}{2(d-2)(d-1)}\left(\partial_{i}^{y^{\prime}} \partial_{a}^{z^{\prime}} \partial_{b}^{z^{\prime}}-\delta_{a i} \partial_{c}^{y^{\prime}} \partial_{b}^{z^{\prime}} \partial_{c}^{z^{\prime}}\right) \frac{w_{0}^{\prime}}{\left[w_{0}^{\prime 2}+\left(\overrightarrow{w^{\prime}}-y^{\prime}\right)^{2}\right]^{d-1}\left[w_{0}^{\prime 2}+\left(\overrightarrow{w^{\prime}}-z^{\prime}\right)^{2}\right]^{d-2}}
\end{gathered}
$$

$A^{\prime}, B^{\prime}$, and $C^{\prime}$ are just $A, B$, and $C$ with the substitutions $y^{\prime} \leftrightarrow z^{\prime}$, and $a \leftrightarrow b$.

We find the following results for the integrals

$$
\begin{gathered}
\int d^{d} w^{\prime} d w_{0}^{\prime}\left(w_{0}^{\prime}\right)^{2 d-4}(A)=\pi^{d / 2} \frac{\left[\Gamma\left(\frac{d}{2}\right)\right]^{3}}{[\Gamma(d)]^{2}} \frac{\delta_{a[i}\left(y^{\prime}-z^{\prime}\right)_{b]}}{\left|y^{\prime}-z^{\prime}\right|^{d}} \\
\int d^{d} w^{\prime} d w_{0}^{\prime}\left(w_{0}^{\prime}\right)^{2 d-4}(B)=\pi^{d / 2} \frac{\left[\Gamma\left(\frac{d}{2}\right)\right]^{3}}{[\Gamma(d)]^{2}} \frac{\delta_{a[i}\left(y^{\prime}-z^{\prime}\right)_{b]}}{\left|y^{\prime}-z^{\prime}\right|^{d}} \\
\int d^{d} w^{\prime} d w_{0}^{\prime}\left(w_{0}^{\prime}\right)^{2 d-4}(C)=-\frac{\pi^{d / 2}}{2(d-1)} \frac{\left[\Gamma\left(\frac{d}{2}\right)\right]^{3}}{[\Gamma(d)]^{2}} \frac{1}{\left|y^{\prime}-z^{\prime}\right|^{d}}\left(\delta_{a b}\left(y^{\prime}-z^{\prime}\right)_{i}+\delta_{i b}\left(y^{\prime}-z^{\prime}\right)_{a}\right. \\
\left.-\delta_{a i}\left(y^{\prime}-z^{\prime}\right)_{b}-\frac{d}{\left|y^{\prime}-z^{\prime}\right|^{2}}\left(y^{\prime}-z^{\prime}\right)_{i}\left(y^{\prime}-z^{\prime}\right)_{a}\left(y^{\prime}-z^{\prime}\right)_{b}\right) .
\end{gathered}
$$

Therefore, putting all of the pieces together, we find

$$
\begin{aligned}
& \int d^{d} w^{\prime} d w_{0}^{\prime}\left(w_{0}^{\prime}\right)^{2 d-4}\left(A+B+C-A^{\prime}-B^{\prime}-C^{\prime}\right) \\
& =\frac{\pi^{d / 2}}{d-1} \frac{\left[\Gamma\left(\frac{d}{2}\right)\right]^{3}}{[\Gamma(d)]^{2}} \frac{1}{\left|y^{\prime}-z^{\prime}\right|^{d}}\left(-\delta_{a b}\left(y^{\prime}-z^{\prime}\right)_{i}+\frac{d}{\left|y^{\prime}-z^{\prime}\right|^{2}}\left(y^{\prime}-z^{\prime}\right)_{i}\left(y^{\prime}-z^{\prime}\right)_{a}\left(y^{\prime}-z^{\prime}\right)_{b}\right) .
\end{aligned}
$$

Which gives the following result for $\mathcal{F}_{i j k}^{(3)}(0, y, z)$

$$
\begin{gathered}
\mathcal{F}_{i j k}^{(3)}(0, y, z)=\kappa(d-2)^{2} \frac{1}{|y|^{2(d-1)}} J_{a j}(y) \frac{1}{|z|^{2(d-1)}} J_{b k}(z) \frac{1}{|t|^{d}}\left(-\delta_{a b} t_{i}+\frac{d}{|t|^{2}} t_{i} t_{a} t_{b}\right) \\
\kappa=\pi^{d / 2}\left(C^{d}\right)^{3} \frac{(d-2)}{(d-1)} \frac{\left[\Gamma\left(\frac{d}{2}\right)\right]^{3}}{[\Gamma(d)]^{2}}, \quad C^{d}=\frac{\Gamma(d)}{2 \pi^{d / 2} \Gamma\left(\frac{d}{2}\right)} .
\end{gathered}
$$

To restore the $x$ dependence we make the replacements $y \rightarrow y-x$ and $z \rightarrow z-x$ and find $\mathcal{F}_{i j k}^{(3)}(0, y-x, z-x)$. This is related to $\mathcal{F}_{i j k}^{(3)}(x, y, z)$ by shift symmetry. Using $t^{2}=$ $(y-z)^{2} /\left[(z-x)^{2}(y-x)^{2}\right]$, we find,

$$
\mathcal{F}_{i j k}^{(3)}(0, y-x, z-x)=\kappa(d-2)^{2} \frac{J_{l j}(y-x)}{|y-x|^{d-2}} \frac{J_{m k}(z-x)}{|z-x|^{d-2}} \frac{1}{|z-y|^{d}}\left(-\delta_{l m} t_{i}+\frac{d}{|t|^{2}} t_{i} t_{l} t_{m}\right) .
$$


Finally, we can express $\mathcal{F}_{i j k}^{(3)}$ in terms of $C_{i j k}$ and $D_{i j k}$ in the following manner:

$$
\begin{aligned}
\frac{t_{l} t_{m}}{t^{2}} & =-\frac{1}{2}\left(J_{l m}(t)-\delta_{l m}\right) \\
J_{l j}(y-x) J_{l m}(t) J_{m k}(z-x) & =J_{l j}\left((y-x)^{\prime}\right) J_{l m}\left((y-x)^{\prime}-(z-x)^{\prime}\right) J_{m k}\left((z-x)^{\prime}\right) \\
& =J_{j k}(y-z) .
\end{aligned}
$$

And we arrive at the following final expression for $\mathcal{F}_{i j k}^{(3)}$ :

$$
\mathcal{F}_{i j k}(x, y, z)=-\kappa \frac{d}{2}\left(D_{j k i}(y, z, x)+\frac{1}{d} C_{j k i}(y, z, x)\right) .
$$

\section{B The embedding space analogs of the conformal tensors $D_{i j k}$ and $C_{i j k}$}

Let $P_{1}, P_{2}, P_{3}$ be points in the embedding $d+2$ dimensional space corresponding to the points $x, y, z$ respectively in the $d$ dimensional physical space:

$$
\begin{aligned}
& P_{x}=\left(1, x^{2}, x^{i}\right) \\
& P_{y}=\left(1, y^{2}, y^{i}\right) \\
& P_{z}=\left(1, z^{2}, z^{i}\right) .
\end{aligned}
$$

The $P_{a},(a=x, y, z)$ are null vectors in the light cone frame in the embedding $d+2$ dimensional space obeying

$$
0=P_{a}^{2}=-P_{a}^{+} P_{a}^{-}+\delta_{i j} P_{a}^{i} P_{a}^{j}
$$

Tensors in the embedding space have corresponding tensors in physical space given by their projection through the Jacobian of the map from the embedding space to the physical space [13]. Consider the following tensors, $I^{M N}$ and $X^{M}$, that appear in equations (1.16) and (1.17) of [16]

$$
\begin{aligned}
I_{x y}^{M N} & =\eta^{M N}-\frac{P_{x}^{M} P_{y}^{N}}{P_{x} \cdot P_{y}} \\
X_{x y}^{M} & =\left(\frac{P_{x}^{M}}{P_{x} \cdot P_{z}}-\frac{P_{y}^{M}}{P_{y} \cdot P_{z}}\right) .
\end{aligned}
$$

Their projections to physical space are the tensors $J_{i j}(x-y)$ and $-2 \tilde{t}_{k}$ given in (3.11) and (3.20) respectively:

$$
\begin{aligned}
J_{i j}(x-y) & =\delta_{i j}-2 \frac{(x-y)_{i}(x-y)_{j}}{(x-y)^{2}} \\
\tilde{t}_{k} & =\left(\frac{(x-z)_{k}}{(x-z)^{2}}-\frac{(y-z)_{k}}{(y-z)^{2}}\right) .
\end{aligned}
$$


Therefore, we can construct the embedding space tensors $\mathcal{D}^{I J K}$ and $\mathcal{C}^{I J K}$ whose projections give $D_{i j k}$ and $C_{i j k}$ as

$$
\begin{aligned}
& \mathcal{D}^{I J K}\left(P_{x}, P_{y}, P_{z}\right)=\frac{-(d-2)^{2}}{2} \frac{1}{P_{x y}^{2}\left(P_{x y}^{2} P_{y z}^{2} P_{z x}^{2}\right)^{(d-2) / 2}} I_{x y}^{I J} X_{x y}^{K} \\
& \mathcal{C}^{I J K}\left(P_{x}, P_{y}, P_{z}\right)=\frac{(d-3)^{2}}{2} \frac{1}{P_{x y}^{2}\left(P_{x y}^{2} P_{y z}^{2} P_{z x}^{2}\right)^{(d-2) / 2}} I_{x z}^{I L} I_{y z}^{L J} X_{x y}^{K},
\end{aligned}
$$

where $P_{x y}=P_{x}-P_{y}$.

Open Access. This article is distributed under the terms of the Creative Commons Attribution License (CC-BY 4.0), which permits any use, distribution and reproduction in any medium, provided the original author(s) and source are credited.

\section{References}

[1] O. Aharony, S.S. Gubser, J.M. Maldacena, H. Ooguri and Y. Oz, Large-N field theories, string theory and gravity, Phys. Rept. 323 (2000) 183 [hep-th/9905111] [INSPIRE].

[2] E. Witten, Anti-de Sitter space and holography, Adv. Theor. Math. Phys. 2 (1998) 253 [hep-th/9802150] [INSPIRE].

[3] I. Heemskerk, J. Penedones, J. Polchinski and J. Sully, Holography from Conformal Field Theory, JHEP 10 (2009) 079 [arXiv:0907.0151] [INSPIRE].

[4] A.L. Fitzpatrick and J. Kaplan, AdS Field Theory from Conformal Field Theory, JHEP 02 (2013) 054 [arXiv:1208.0337] [INSPIRE].

[5] A.L. Fitzpatrick, E. Katz, D. Poland and D. Simmons-Duffin, Effective Conformal Theory and the Flat-Space Limit of AdS, JHEP 07 (2011) 023 [arXiv: 1007.2412] [InSPIRE].

[6] R. Sundrum, From Fixed Points to the Fifth Dimension, Phys. Rev. D 86 (2012) 085025 [arXiv:1106.4501] [INSPIRE].

[7] D.Z. Freedman, S.D. Mathur, A. Matusis and L. Rastelli, Correlation functions in the $C F T_{d} / A d S_{d+1}$ correspondence, Nucl. Phys. B 546 (1999) 96 [hep-th/9804058] [INSPIRE].

[8] G. Chalmers, H. Nastase, K. Schalm and R. Siebelink, $R$ current correlators in $N=4$ super Yang-Mills theory from anti-de Sitter supergravity, Nucl. Phys. B 540 (1999) 247 [hep-th/9805105] [INSPIRE].

[9] S. Lee, S. Minwalla, M. Rangamani and N. Seiberg, Three point functions of chiral operators in $D=4, N=4 S Y M$ at large-N, Adv. Theor. Math. Phys. 2 (1998) 697 [hep-th/9806074] [INSPIRE].

[10] D.Z. Freedman, G. Grignani, K. Johnson and N. Rius, Conformal symmetry and differential regularization of the three gluon vertex, Annals Phys. 218 (1992) 75 [hep-th/9204004] [INSPIRE].

[11] D. Anselmi, D.Z. Freedman, M.T. Grisaru and A.A. Johansen, Nonperturbative formulas for central functions of supersymmetric gauge theories, Nucl. Phys. B 526 (1998) 543 [hep-th/9708042] [INSPIRE].

[12] D.M. Hofman and J. Maldacena, Conformal collider physics: Energy and charge correlations, JHEP 05 (2008) 012 [arXiv: 0803.1467] [INSPIRE]. 
[13] M.S. Costa, J. Penedones, D. Poland and S. Rychkov, Spinning Conformal Correlators, JHEP 11 (2011) 071 [arXiv:1107.3554] [INSPIRE].

[14] S. Giombi, S. Prakash and X. Yin, A Note on CFT Correlators in Three Dimensions, JHEP 07 (2013) 105 [arXiv: 1104.4317] [INSPIRE].

[15] H. Osborn and A.C. Petkou, Implications of conformal invariance in field theories for general dimensions, Annals Phys. 231 (1994) 311 [hep-th/9307010] [INSPIRE].

[16] M.F. Paulos, Towards Feynman rules for Mellin amplitudes, JHEP 10 (2011) 074 [arXiv:1107.1504] [INSPIRE]. 